\title{
Análisis Económico de Medidas de Política Agrícola y Ventajas Comparativas de la Producción de Trigo en Dos Áreas de Guatemala ${ }^{1}$
}

\author{
David E. Castañon Orozco ${ }^{2}$, Gustavo E. Sain ${ }^{3}$ y Juan Carlos Martínez ${ }^{4}$
}

\section{INTRODUCCION}

La producción agropecuaria guatemalteca se caracteriza por ser el soporte fundamental de la economía nacional. En el sector agropecuario guatemalteco, coexisten varias tipologías de producción que pueden agruparse fundamentalmente en dos grandes sectores. Por un lado existe un sector moderno que produce para la exportación: café, algodón, banano, caña de azúcar, carne y cardamomo, que hace uso de nuevas tecnologías, tienen acceso al crédito y opera en grandes fincas de las regiones tropicales de la costa sur del país. Este sector, a su vez, demanda fuertes cantidades de mano de obra de manera estacional, principalmente para levantar las cosechas, requiriendo para el efecto mano de obra temporal y migratoria que se desplaza de las altiplanicies a la costa. En estas altiplanicies es donde se localiza la producción del trigo nacional. Este sector que se podría identificar como moderno o comercial, coexiste con un sector que podría caracterizarse como en rezago, el cual produce principalmente rubros para el consumo interno: maíz, frijol, trigo, frutas y hortalizas, hace uso de limitadas innovaciones técnicas, la producción se realiza en pequeña escala y tiene dificultades para la obtención de créditos.

A nivel de rubros específicos el café continua siendo el principal producto agrícola del país, tanto por su participación en el Producto Interno Bruto (PIB) como por la generación de divisas. Este cultivo ha mantenido una relativa estabilidad dada las cuotas establecidas por el Acuerdo Internacional del Café.

Dentro de los cultivos alimenticios se destacan el maíz y el frijol, cuya producción ha ido incrementando aunque se ha tenido que recurrir a importaciones para satisfacer la demanda interna. En cuanto a arroz se estima que el país ha ido alcanzando autosuficiencia a través de una generalización de innovaciones técnicas con agricultores grandes de producción intensiva.

En el caso del trigo, en Guatemala existen actualmente once áreas homogéneas de producción de trigo que abarcan alrededor de 30,000 hectáreas. Esta gran diversidad se explica parcialmente por la enorme variabilidad microclimática, y étnica predominante en el área de producción.

De estas once áreas o zonas, las principales en términos de volumen de producción corresponden al valle de Quetzaltenango y a la parte alta de Chimaltenango; ambas zonas presentan características distintas en lo que al sistema de producción de trigo le concierne. En el valle de Quetzalte- nango, el cultivo de trigo compite en el uso de la tierra con el cultivo de maíz y en muy pequeña escala con la papa, mientras que en Chimaltenango el sistema ha venido sustituyendo el cultivo de trigo por crucíferas en general y elbrócoli en particular.

La importancia del brócoli dentro de la economía guatemalteca no debe ser despreciada ya que mientras el trigo es un cultivo netamente de importación, las exportaoiones de brócoli se han incrementado sustancialmente durante la década de los 80 (Cuadro 1).

En el caso de maíz sucede algo especial; este cereal es el principal alimento de la población guatemalteca cuyos gustos de consumo estan arraigados en el grano de color blanco.

Cuadro 1. Exportaciones de Brócoli. Guatemala 1983-1988

\begin{tabular}{crc}
\hline AÑOS & $\begin{array}{r}\text { CANTIDAD } \\
(\mathrm{kgs})\end{array}$ & $\begin{array}{r}\text { VALOR } \\
\text { (Quetzales) }\end{array}$ \\
\hline 1983 & $1,421,112$ & $1,163,474$ \\
1984 & 119,257 & 40.195 \\
1985 & $1,447,235$ & 837,687 \\
1986 & $5,974,130$ & $2.598,076$ \\
1987 & $12,389,432$ & 7.988 .142 \\
1988 & $\mathbf{5 7 , 6 2 7 , 4 3 0}$ & $17.732,697$ \\
\hline
\end{tabular}

Fuente: Departamento de Cuarentena Vegetal, DIGESA. Guatemala

Este tipo de grano es el que más se produce localmente, mientras que el grano que se comercializa internacionalmente es de color amarillo cuya producción local no es suficiente para abastecer a la industria local que elabora concentrados para alimentación animal. Esto obliga a que en ocasiones se deba recurrir al mercado internacional para abastecerse de maíz de color amarillo.

En materia de política de precios agrícolas, el estado ha ido liberando el control de precios para la gran mayoría de

${ }^{1}$ Esta es una versión editada del trabajo presentado en la XXXVI Reunión Anual del Programa Cooperativo Centroamericano para el Mejoramiento de Cultivos Alimenticios (PCCMCA). San Salvador, 26-30 de marzo de 1990.

2 Consultor CIMMYT. Previamente en la Sección de Socioeconomía del ICTA. Guatemala.

${ }^{3}$ Economista Regional para Centro América y El Caribe del CIMMYT

${ }^{4}$ Research Fellow in Public Policy and Managemment. John F. Kennedy School of Goverment, Harvard University. A nteriormente Economista Regional para Centro América y El Caribe del CIMMYT.

Publicado en Agronomía Mesoamericana. Vol. 2 (1991) 
productos y la intervención en la política de sustentación de precios para maíz y frijol ha disminuido. Actualmente ónicamente se encuentra sujeto a control de precios 14 productos, entre ellos se encuentra el trigo producido localmente y los productos derivados de la industria del trigo. Siendo en este caso un producto sensible dada la presión que ejercen sectores urbanos por obtoner pan a bajo precio.

\section{DEFINICION, IMPORTANCIA, Y OBJETIVOS DEL ESTUDIO}

La asignación de recursos económicos en términos de su eficiencia económica ha cobrado mayor importancia en los últimos años tanto para los productores privados como para el Gobierno de Guatemala. Esta situación cobra aún más relevancia debido a los ajustes de tipo estructural que realizan en el país tratando en lo posible de que el estado intervenga menos en la economía. De esta manera se han venido planteando la necesidad de conocer no solamente la rentabilidad privada sino también la rentabilidad social de las distintas actividades agropecuarias, con el fin de poder determinar la correcta asignación de recursos en términos de su eficiencia económica.

En el caso de la producción de trigo nacional se hace necesario realizar un análisis que permita orientar la toma de decisiones, no solo en el campo de la política económica sino también en las perspectivas de investigación y transferencia de tecnología en que se ven inmersos organismos privados, públicos y de cooperación internacional.

El presente trabajo fue planteado con dos objetivos básicos. En primer lugar el de dar a conocer la eficiencia económica privada y social de los sistemas de producción de trigo predominantes en dos áreas en Guatemala: el Valle de Quetzaltenango, y la parte alta de Chimaltenango. El segundo objetivo propuesto es el de analizar los impactos de las políticas agrícola, estableciendo o no la existencia de distorsiones que limiten o favorezcan la producción de trigo en Guatemala.

\section{METODOLOGIA}

Para estimar los efectos de las medidas de política agrícola sobre los estímulos o incentivos a la producción se usan dos coeficientes: el Coeficiente de Protección Nominal (CPN) y el Coeficiente de Protección Efectivo (CPE).

El CPN es la más simple y quizás la más usada de las medidas de distorsiones de políticas. Está definida como la relación entre el precio doméstico de un producto y su precio de frontera (precio mundial equivalente). Es decir, para un producto i, el CPN se expresa mediante la fórmula siguiente:

$$
\text { (1) } \mathrm{CPN}_{\mathrm{i}}=\frac{\mathrm{p}_{\mathrm{i}}^{\mathrm{d}}}{\mathrm{p}_{\mathrm{i}}^{\mathrm{m}}}
$$

El CPN mide el impacto del conjunto de medidas de intervención del gobierno y distorsiones en los mercados sobre la asignación de recursos en la actividad i.

El CPN puede ser mayor o menor que uno, resultando en distorsiones o interacciones que favorecen o desfavorecen a la producción doméstica del bien en cuestión.

Una segunda medida de los impactos de las intervenciones y distorsiones en los mercados está dada por el coeficiente de protección efectiva (CPE). El CPE mide interferencias tanto en los mercados del producto final como en los mercados de los in sumos utilizados en la elaboración del producto final.

El CPE para el producto i es definido como la relación entre el valor agregado estimado a precios domésticos $\mathrm{VA}_{\mathrm{i}}{ }^{\mathrm{d}}$ y el valor agregado estimado bajo el supuesto de libre comercio (aprecios mundiales) $\mathrm{VA}_{\mathrm{i}}^{\mathrm{m}}$ :

(2)

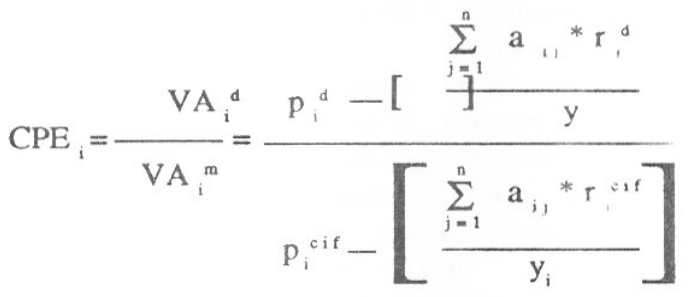

donde:

CPEi $=$ Coeficiente de protección efectiva para el producto "i"; $\mathrm{a}_{\mathrm{ij}}=$ cantidad por unidad de superficie del insumo j-ésimo empleado en la producción del producto $\mathrm{i} ; \mathrm{r}_{\mathrm{j}}^{\mathrm{d}}=$ precio interno del j-ésimo insumo que se moviliza en los mercados internacionales; $r_{j}{ }_{j}^{\text {cif }}=$ precio cif del $\mathrm{j}$-ésimo insumo que se moviliza en los mercados internacionales, valorado a precios de frontera y convertido a la tasa de cambio corregi$\mathrm{da} ; \mathrm{y}_{1}=$ rendimiento obtenido del producto "i" $; \mathrm{p}_{1}{ }^{\mathrm{d}}, \mathrm{p}_{\mathrm{i}}{ }^{\mathrm{cif}}=$ precio interno y de frontera respectivamente.

Si el CPE es mayor que 1, habrá incentivos para usar más in sumos en la industria de los que se usarían bajo el libre comercio, mientras que si el CPE está por debajo de 1 habrá incentivos para no usar (sacar) insumos de la actividad y el nivel estará por debajo de lo que se usaría bajo libre comercio (eficiencia).

Finalmente, la tercera medida que se considera en este estudio es el coeficiente denominado Costos de los Recursos Domésticos (CRD).

Este coeficiente intenta medir la eficiencia relativa de producir un bien internamente en comparación con la alternativa de importarlo. En otras palabras, mide la eficiencia relativa de las alternativas de producción en el uso de los 
recursos domésticos para ganar (o ahorrar) una unidad de divisa. En este sentido mide la existencia o no de ventajas comparativas del país en producir domésticamente el bien.

Con este criterio un país tiene ventajas comparativas en la producción del bien si el gasto en recursos domésticos (tierra, trabajo y capital) medidos en términos de precios internacionales es menor que el costo doméstico por unidad de divisas necesarias para importar el bien. Consecuentemente, si el costo es mayor el país estaría mejor importando el bien, es decir, no tendría ventajas comparativas.

El CRD se computa de acuerdo a la fórmula siguiente:

(3) $\mathrm{CRD}=$

$$
\sum_{j=k}^{n} a_{i j} * r_{j}{ }_{j}^{s}
$$

$$
P_{i}^{c i f}-\left[\sum_{j=1}^{k-1} a_{i j} * r_{j}{ }^{c i f}\right]
$$

donde:

$\mathrm{a}_{\mathrm{ij}}=$ coeficiente técnico que refleja la cantidad de insumo " $\mathrm{j}$ " usado en la producción del bien" $\mathrm{i}$ ". Notesé que existen $\mathrm{j}=1 \ldots \mathrm{k}-1$ insumos comerciables, $\mathrm{y} \mathrm{k}-\mathrm{n}$ insumos no comerciables. $\mathrm{r}_{\mathrm{j}}^{\mathrm{g}}=$ costo de oportunidad del $\mathrm{j}$-ésimo recurso o bien primario no comerciable; $r_{j}{ }^{\text {eif }}=$ precio internacional del j-ésimo in sumo comerciable.

Para el cálculo correcto de estos indicadores se debe tener en cuenta varios aspectos metodológicos.

En primer lugar se debe escoger un punto de comparación dentro de la cadena de comercialización. El precio doméstico en estos casos es el precio observado en el punto de comparación. Si no existiere un mercado o precios observados entonces el precio doméstico se calcula como el precio recibido por los productores ajustados por los costos de mercadeo desde la finca hasta el punto de comparación. En el caso de los precios mundiales estos también deben ajustarse por los costos de comercialización desde el puerto de entrada (salida) hasta el punto de comparación.

Un segundo aspecto importante resulta del hecho de que el precio mundial y el precio doméstico deben ser expresados en la misma moneda, y para ello se necesita una tasa de cambio. Usualmente la tasa de cambio oficial (TCO) es una tasa que sobreevalúa la moneda local no reflejando de esta manera el costo de oportunidad o precio de escasez de las divisas. Por ello resulta conveniente usar una tasa de cambio ajustada que refleje mejor el verdadero precio social de las divisas.

Para el cálculo de la rentabilidad social, los in sumos y productos deben ser valuados a los precios de eficiencia to- mando como referencia los precios internacionales para aquellos bienes y servicios considerados como comerciables. Aquellos que no lo fueran, son descompuestos en sus componentes comerciables y recursos domésticos y se procede a su valuación. Los recursos domésticos, tierra, trabajo, y capital son valuados según sus precios sombra o costos de oportunidad.

En este trabajo, el cálculo de los precios de eficiencia se realizó a través de la utilización de factores de conversión por producto. Esto es, factores que permiten convertir los precios de mercado en la finca a precios de eficiencia.

Para el presente estudio, se decidió estimar inicialmente un factor de conversión estándar (FCE), para convertir el valor de grandes agregados de materiales de origen nacional a precios en la frontera y un factor de conversión para la mano de obra, llamado también precio sombra de la mano de obra.

El FCE se calculó como la relación entre el valor agregado de exportaciones e importaciones a precios de frontera (FOB y CIF) y el valor de estos mismos bienes en precios domésticos:

(4) $\mathrm{FCE}=(\mathrm{X}+\mathrm{M}) /(\mathrm{X}-\mathrm{Tx})+(\mathrm{M}+\mathrm{Tm})$

donde: X y M representan el valor de las exportaciones e importaciones respectivamente evaluadas a precios internacionales (FOB y CIF); y Tx y Tm representan el valor de todos los impuestos afectando a las .exportaciones e importaciones respectivamente.

Posteriormente se estimaron factores de conversión para el diesel, transporte y mercadeo, ya que ellos forman parte de la estimación de los factores de conversión para los demás bienes y servicios. Finalmente se estimó una serie de factores de conversión para productos agropecuarios, agroquímicos y bomba de asperjar, a fin de cubrir la mayoría de bienes y servicios contemplados en el análisis.

\section{RESULTADOS}

\section{l. El factor de conversión estandar (FCE)}

La economía de Guatemala se ha caracterizado por ser de tipo abierta, con una relativa intervención del estado. En efecto, con base a la serie histórica de 1970-85 de los movimientos del comercio exterior, se estimó el coeficiente de conversión estandard con base a la fórmula (4). Para ello, se obtuvo información oficial sobre el valor de las importaciones, en precios CIF a la tasa de cambio oficial, las exportaciones, precios FOB a la tasa de cambio oficial, asi como del valor de los aranceles y demás impuestos para el período 1970-85.

El valor de esta relación para el período 70-85 oscila entre 0.95 y 1 aproximadamente, lo que indica la gran apertura de la economía guatemalteca. Con base en lo anterior se 
decidió adoptar un valor para el FCE de 0.97, que se estima apropiado para convertir precios internos de grandes agregados de materiales de origen nacional a precios en la frontera.

\section{Los precios privados y de eficiencia}

\subsection{Aspectos generales}

Para la obtención de los precios privados se consultó la información recopilada por la disciplina de Socioeconomía Rural del Instituto de Ciencia y Tecnología Agrícolas ICTA, a través de los llamados Registros Económicos de Producción, actualizada por informantes de la Dirección General de Servicios Agrícolas - DIGESA- y consultas directas a productores y tiendas agropecuarías efectuadas en las zonas de producción con referencia al año de 1988.

Para las estimaciones del precio de eficiencia se procedió a diferenciar los bienes transables y no transables. La estimación del precio de cuenta de un bien transable internacionalmente depende de tres factores: $\mathrm{Si}$ el bien en cuestión es exportable o importable, si la cantidad consumida o vendida afecta el precio vigente y del punto de comercialización.

En cuanto al punto de comercialización, en este estudio se adoptó a la finca como punto de referencia, por tanto todos los precios utilizados se dan a este nivel.

Por su parte, los bienes no transables son los que coo'csponden propiamente a la economía interna, siendo en su oportunidad ajustados por el factor estandard.

Para los precios CIF y FOB se utilizó la moneda local a la tasa de cambio oficial vigente en 1988 (Q. $2.70 \mathrm{x}$ $\$ 1.00)$. A este tipo de cambio se registran los datos de importación y exportación de productos agrícolas en el Departamento de Cuarentena Vegetal de DIGESA, fuente básica para la presente información.

Cuando los productos se valoran en la finca, el precio respectivo incluye provisiones para cubrir costos de transporte, impuestos y gastos de comercialización, bien en la rnta hacia la finca o de esta hacia los puertos de salida del país.

Los rendimientos usados en el análisis fueron de $2.3 \mathrm{y}$ 2.7 t/ha para el trigo en la región del Valle de Quetzaltenango, y en la parte alta de Chimaltenango respectivamente. Para el maíz, el rendimiento fue de $6.5 \mathrm{t} /$ ha, mientras que para el brócoli fue de $11.5 \mathrm{t} / \mathrm{ha}$. Estos valores son promedios de aquellos obtenidos por los agricultores de las áreas en estudio y reflejan un nivel tecnológico modal.

\section{La matriz de análisis de política}

Una forma conveniente de sintetizar la información de los efectos de las políticas la suministra la matriz de análisis de política (Monke y Pearson, 1989). En ellas se organizan los presupuestos de cada actividad evaluada tanto a precios privados como sociales. Una tercera fila computa las divergencias existentes entre ambos lo que permite visualizar rápidamente los impactos de políticas y otras distorsiones sobre los diferentes elementos del presupuesto.

En el Cuadro 2 se presenta la matriz de análisis de política para los cultivos maíz y trigo en el Valle de Quetzaltenango.

Examinando la columna de beneficios resulta evidente que la siembra de maíz es más beneficiosa que la de trigo tanto en términos privados como sociales. Esto refleja el menor uso de insumos por parte del cultivo de maíz lo que se deriva en una mayor rentabilidad.

Cuadro 2. Matriz de Análisis de Política para Trigo y Maíz en el Valle Quetzaltenango, Guatemala

(a) Análisis por Hectárea

\begin{tabular}{c} 
Ingresos \\
\cline { 2 - 3 } Insumos $\quad$ Factores
\end{tabular}

\begin{tabular}{lrrrr}
$\begin{array}{l}\text { A precios } \\
\text { privados } \\
\text { Trigo }\end{array}$ & 1196.00 & 367.88 & 821.30 & 6.80 \\
Maiz & 1980.00 & 282.40 & 1311.50 & 386.10 \\
& & & & \\
A precios & & & & \\
sociales & 1034.66 & 320.13 & 753.66 & -39.13 \\
$\begin{array}{l}\text { Trigo } \\
\text { Maiz }\end{array}$ & 1920.60 & 236.24 & 1200.88 & 483.46 \\
& & & & \\
$\begin{array}{l}\text { Divergencias } \\
\text { Trigo }\end{array}$ & 161.34 & 47.74 & 67.64 & 45.94 \\
Maiz & 59.40 & 46.15 & 110.61 & -97.36 \\
\hline
\end{tabular}

(b) Análisis por Tonelada Métrica

\begin{tabular}{lrrrr}
\hline & Ingresos & \multicolumn{2}{c}{ Costos } & Beneficios \\
\cline { 3 - 4 } & & Iinsumos & Factores & \\
\hline A precios & & & & \\
privados & & & 357.08 & 2.96 \\
Trigo & 520.00 & 159.95 & 291.44 & 85.80 \\
Maiz & 440.00 & 62.75 & & \\
& & & & \\
A precios & & & & 17.01 \\
Sociales & & & & \\
Trigo & 449.85 & 139.19 & 266.864 & 107.43 \\
Maiz & 426.80 & 52.49 & & \\
& & & & \\
\hline
\end{tabular}

En la fila de divergencias, es importante destacar la magnitud en la columna de ingresos. Aunque en ambos casos es positiva los valores para el trigo están muy por ambas que aquellos para el maíz reflejando un nivel de protección vía precio del trigo de aproximadamente un $16 \%$. A nivel 
de beneficios por unidad de tierra, la divergencia positiva para el trigo indica que el balance del efecto de todas las poHticas dan incenti vos adicionales a los previstos por los precios mundiales para la producción de trigo mientras que ocurre lo contrario para el maíz.

En el caso del trigo, la relación de factores primarios respecto a ingresos y costos privados es de 0.99 mientras que para el maíz es de 0.77 , lo que significa que para el maíz es más competitivo el uso de factores valorados a precios de mercado.

Por otra parte, la relación costo de los recursos domésticos, CRD arroja valores de 1.05 para el trigo y 0.71 para el maíz. Estas cifras expresan la eficiencia del uso de recursos locales para la producción de maíz. En este caso, el Valle de Quetzaltenango tiene ventaja comparativa para la producción de maíz, mientras que para el trigo el indicador mayor que 1 esta mostrando que es un poco más alto el uso de recursos domésticos contra las posibilidades de importar el producto. En otro sentido, el costo de los recursos domésticos usados en la producción de maíz es menor que el valor de los recursos domésticos que se ganan sustituyendo importaciones de trigo.

El coeficiente de rentabilidad relaciona los beneficios pnvados con los benet1cios sociales y da una medida del grado en que las transferencias netas causan divergencias entre la utilidad privada y social. En el caso del trigo el coeficiente es negativo dada la transferencia de beneficios de la sociedad hacia el productor para la recuperación de sus costos de producción, en tanto que para el maíz es inferior al, colocándolo como un producto con mucha compctilividad.

Con el apoyo de la información de la matriz. se han realizado los cálculos de los coeficientes de protección nominal y protección efectiva. Al comparar los datos se observa que el coeficiente de protección nominal del trigo es de 1.15 y el efectivo de 1.16; mientras que para el maíz es de 1.03 y 1.01 respectivamente. Esta situación refleja que para el trigo los agricultores están recibiendo una protección tanto nominal como efectiva por parte de las medidas de poHtica del estado, mientras que para el maíz cuyos coeficientes están muy próximos a 1 muestran que no existen distorsiones importantes que estimulen su producción.

El Cuadro $3 \mathrm{~m}$ uestra la matriz de análisis de política para el área de la parte alta de Chimaltenango, se aprecia que el cultivo de trigo genera beneficios positivos a precios privados, mientras que a precios sociales se observa pérdida. Mientras que, el brócoli genera beneficios positivos tanto a nivel privado como social. Aún así, se debe notar que los beneficios sociales derivados de la producción de brócoli se reducen en más de $300 \%$ respecto al nivel privado debido al uso intensivo de componentes importados.

En la fila de divergencias, el patrón respecto al trigo es similar a aquél de Quetzaltenango, mientras que para el brócoli la divergencia en los beneficios es bastante alta, aún que
Cuadro 3. Matriz de Anślisis de Política para Trigo y Brócoli en el Area de Chimaltenango, Guatemala

(a) Análisis por Hectárea.

\begin{tabular}{lrrrr}
\hline & Ingresos & \multicolumn{2}{c}{ Costos } & \multirow{2}{*}{ Beneficios } \\
\cline { 3 - 4 } & & Insumos & Factores \\
\hline A precios & & & & \\
privados & & & & \\
Trigo & 1404.00 & 375.32 & 980.56 & 48.11 \\
Brócoli & 5512.50 & 2373.10 & 2406.51 & 732.88 \\
& & & & \\
A precios & & & & \\
sociales & & & & \\
Trigo & 1214.60 & 332.01 & & \\
Brócoli & 4337.35 & 1961.20 & 2205.06 & 171.08 \\
Divergencias & & & & \\
Trigo & 189.39 & 43.30 & 75.89 & 70.19 \\
Brócoli & 1175.15 & 411.89 & 201.4 & 561.80 \\
\hline
\end{tabular}

(b) Análisis por Tonelada Métrica.

\begin{tabular}{|c|c|c|c|c|}
\hline & \multirow[t]{2}{*}{ Ingresos } & \multicolumn{2}{|c|}{ Costos } & \multirow[t]{2}{*}{ Beneficios } \\
\hline & & linsumos & Factores & \\
\hline \multicolumn{5}{|c|}{$\begin{array}{l}\text { A precios } \\
\text { privados }\end{array}$} \\
\hline Trigo & 520.00 & 139.00 & 363.17 & 17.81 \\
\hline Brócoli & 490.00 & 210.94 & $2 \longdiv { 3 . 9 1 }$ & 6514 \\
\hline \multicolumn{5}{|c|}{$\begin{array}{l}\text { A precios } \\
\text { sociales }\end{array}$} \\
\hline Trigo & 449.85 & 122.96 & 335.06 & -8.17 \\
\hline Brócoli & 385.54 & 174.32 & 196.00 & 15.20 \\
\hline \multicolumn{5}{|c|}{ Divergencias } \\
\hline Trigo & 70.14 & 16.04 & 28.11 & 25.99 \\
\hline Brócoli & 104.45 & 36.61 & 17.90 & 49.93 \\
\hline
\end{tabular}

no en extremo si se compara con el maíz de Quetzaltenango.

En términos de rentabilidad el brócoli presenta una rentabilidad del $15.3 \%$, mientras que para el trigo esta es de $3.5 \%$. La relación de costos privados es de 0.95 para trigo y 0.77 para brócoli, lo que indica que es más competitivo el uso de factores en el brócoli que para el trigo. La relación del costo de los recursos domésticos CRD, da valores de 1.02 y 0.93 para trigo y brócoli respectivamente; expresando el coeficiente la eficiencia en el uso de los recursos locales para el brócoli. Al igual que para el caso de Quetzaltenango el coeficiente de rentabilidad en trigo es negativo dada la transferencia de beneficios de los consumidores hacia el productor; mientras que para el brócoli este indicador es positivo (4.2\%).

Al referirse al cálculo de la protección nominal y efectiva en estos cultivos se observa que los coeficientes son muy parecidos a los detectados en Quetzaltenango con respecto al cultivo de trigo. Estos coeficientes son de 1.15 para la protección nominal y de 1.16 para la protección 
efectiva; mostrando la protección que prevalece en el cultivo a nivel del país por parte de las políticas estatales. En lo que se refiere a brócoli el coeficiente de protección nominal es de 0.92 y la efectiva es de 0.77 ; esta situación muestra la desprotección del cultivo. derivado principalmente del alto contenido de insumos que se encuentran sujetos a significativos derechos arancelarios de importación. Por otro lado. de la relación entre los costos de los recursos domésticos y el coeficiente de protección efectiva indican que este cultivo es eficiente, pero a su vez, muestra que la política gubernamental está explotando la ventaja comparativa que representa el mantenimiento de precios bajos para la mano de obra, pudiéndose inferir también las ventajas de precios bajos para el productor que favorece a las agro industrias de congelados, quienes en última instancia son las que mantienen los vínculos comerciales con el exterior.

\section{CONCLUSIONES Y RECOMENDACIONES}

1 La metodología pone en evidencia la relación de precios de eficiencia entre la producción nacional y el mercado mundial, de donde los coeficientes permiten medir la ventaja comparativa que posee el país respecto a los intercambios internacionales y el uso alternativo de la tierra y de los demás faclores de la producción.

2. En el caso del Valle de Quetzaltenango, resulta que el maíz es el cultivo con mayores ventajas respecto al trigo; este último se encuentra protegido por las políticas estatales permitiendo tanto una protección nominal como efectiva que permite rentabilidad a precios privados pero no a nivel de precios sociales.

3. Para permitir reducir la protección del trigo será necesario incrementar los rendimientos actuales sobre la base de una estabilización en los costos de producción. En estas circunstancias el mejoramiento genético sigue constituyendo la opción en materia de innovación tecnológica.

4. En el caso de la parte alta de Chimaltenango, de hecho el trigo ha sido paulatinamente desplazado por las hortalizas. El análisis respecto al brócoli indica las ventajas tanto a precios privados como sociales que representa este cultivo.

5. El brócoli es un cultivo que se encuentra desprotegido en términos de política estatal, aunque en la práctica hay una tendencia a favorecer la exportación de productos no tradicionales, que como en este caso tienen ventaja comparativa para el país. No obstante, este cultivo demanda una fuerte cantidad de componente transable importado, de manera que las opciones de incrementar las ventajas del cultivo debieran orientarse a hacer más eficiente el uso de insumos

\section{BIBLIOGRAFIA}

BYERLEE, Derek; LONGMIRE,J. 1986. Ventaja Comparativa y Política Agrícola de la Producción de Trigo en Zonas Irrigadas y de Secano en México. Documento de trabajo No. 01186. Programa de Economía-CIMMYT, México. D.F.

MONKE. E.: PEARSON,S. 1989. The Policy Analysis Matrix, Baltímore: Johns Hopkiins University Press.

NUILA MELENDEZ, ALMA SONIA. 1989. Análisis Económico de Medidas de Política Agrícola y Ventajas Comparativas del sistema de relevo Maíz/Frijol para el área de Opico-Quezaltepeque. El Salvador Tesis de Maestría en Economía Agrícola. Universidad de Puerto Rico 1989.

SAIN, Gustavo; MARTINEZ, J.C. 1989. Política de Precios para Granos Básicos en Latinoamerica. Trabajo Presentado en la XXXV Reunión Anual del Programa Cooperativo Centroamericano para e1 Mejoramiento de Cultivos Alimenticios (PCCMCA). San Pedro Sula. Honduras. 3-7 Abril de 1989, 